\begin{tabular}{|c|l|}
\hline Title & $\begin{array}{l}\text { Spectral and correlation analyses of the verapamil-induced conversion of ventricular fibrillation to tachycardia in } \\
\text { isolated rat hearts }\end{array}$ \\
\hline Author(s) & Kawahara, Koichi; Takase, Marei; Y amauchi, Y oshiko; Kimura, Hiroyuki \\
\hline Citation & $\begin{array}{l}\text { Journal of Electrocardiology, 37(2), 89-100 } \\
\text { https://doi.org/10.1016/.jelectrocard.2004.01.006 }\end{array}$ \\
\hline Issue Date & 2004-04 \\
\hline Doc URL & http://hdl.handle.net/2115/5932 \\
\hline Rights(URL) & http://www.elsevier.com/wpsfind/ournaldescription.cws_home/623308/description \\
\hline Type & article (author version) \\
\hline File Information & JE37-2.pdf \\
\hline
\end{tabular}

Instructions for use 


\title{
Spectral and correlation analyses of the verapamil-induced conversion of ventricular fibrillation to tachycardia in isolated rat hearts.
}

\author{
Koichi Kawahara, PhD, Marei Takase, MS, Yoshiko Yamauchi, MS, and Hiroyuki
}

Kimura

Laboratory of Biomedical Control, Research Institute for Electronic Science, Hokkaido

University, Sapporo 060-0812, JAPAN

Short Title: verapamil-induced conversion of VF to VT

\section{Address correspondence to:}

Koichi Kawahara, Ph D.

Professor of the Laboratory of Biomedical Control,

Research Institute for Electronic Science,

Hokkaido University,

Sapporo 060-0812,

JAPAN

TEL \& FAX: +81-11-706-2893

E-mail: kawahara@bmc.es.hokudai.ac.jp 


\section{Abstract}

Ventricular tachycardia (VT) is considered to be the most common precursor of ventricular fibrillation (VF). However, the mechanisms underlying the transition from VT to VF remain unclear. Here we investigated whether and how perfusion of the heart with verapamil, a blocker of L-type calcium channels, changed the macro-dynamics of the heart between VT and VF. The experiments were performed using Langendorff perfused isolated rat hearts, in which left ventricular pressure (LVP) and left ventricular cardiomyogram (LVCMG) were measured. Sustained VT or VF was induced by burst pacing of the left ventricular muscles. During sustained VF, verapamil perfusion resulted in the conversion of VF to VT. A cross-correlation analysis between LVCMG and LVP revealed that the correlation coefficient was small during VF, but became larger during VT. This study demonstrated that verapamil treatment converted sustained VF to VT, and characterized the changes in macro-dynamics of the heart associated with the transition.

Key words: ventricular fibrillation, ventricular tachycardia, VT/VF transition, calcium channels, verapamil 


\section{Introduction}

Ventricular fibrillation (VF) and reentrant ventricular tachycardia (VT) are the major immediate causes of sudden cardiac death (1). Whereas VT is considered a rapid but well organized process, VF has been described as turbulent cardiac electrical activity, resulting from the random and aperiodic propagation of multiple independent wavelets throughout the cardiac muscle (2). Clinical studies have shown that VF is almost always preceded by VT of variable duration, from a few to many beats $(3,4)$. Also, in isolated rat hearts, spontaneous transition from VT to VF is frequently observed during reperfusion after global ischemia (5). However, the manner in which VT converts to VF, or vice versa is not yet fully understood despite more than a century of intensive study.

A number of previous studies have revealed that intracellular $\mathrm{Ca}^{2+}$ overload predisposes the myocardium to abnormal electrical activities promoting VF $(6,7)$. In addition, several $\mathrm{Ca}^{2+}$ channel antagonists have been shown to prevent VF induced by myocardial ischemia in animals $(8,9)$, as well as to reduce cardiac mortality in patients recovering from a myocardial infarction $(10,11)$. Merillat et al. (12) reported that an increase in cytosolic, global $\mathrm{Ca}^{2+}$ per se seems unnecessary for the initiation and maintenance of VF, and that an increase of $\mathrm{Ca}^{2+}$ influx 
through L-type $\mathrm{Ca}^{2+}$ channel (slow $\mathrm{Ca}^{2+}$ channel) is essential. A previous report by Watanabe and Uchida (13) demonstrated in isolated rabbit hearts that rapid ventricular stimulation in the presence of verapamil, an antagonist of L-type $\mathrm{Ca}^{2+}$ channel, resulted in the induction of sustained monomorphic VT, not VF. In a recent study by Samie et al. (14), verapamil perfusion in Langendorff-perfused rabbit hearts converts burst pacing-induced VF to VT. They postulated that verapamil-induced VF-to-VT conversion is most likely due to a reduction in the frequency rotors and a decrease in wavefront fragmentation that lessens fibrillatory propagation. We have recently demonstrated that perfusion of isolated hearts with ruthenium red (RR), a blocker of mitochondrial $\mathrm{Ca}^{2+}$ uptake, reversibly converts sustained VF to VT, and that such RR-induced conversion of VF to VT is antagonized by co-treatment with S(-)-Bay K8644, an activator of L-type $\mathrm{Ca}^{2+}$ channels, suggesting that the inactivation of L-type $\mathrm{Ca}^{2+}$ channels is responsible for the RR-induced effect on the macro-dynamics of hearts (15). All these findings have led to an idea that the change in the activity of L-type $\mathrm{Ca}^{2+}$ channels is crucially involved in the transition between VT and VF. However, there still remains uncertainly as to what mechanisms are critically involved in the verapamil-induced VF-to-VT conversion. In addition, the changes in the macro-dynamics of hearts during 
verapamil-induced VF-to-VT conversion have not yet been well characterized.

In this study, using isolated rat hearts, we investigated whether and how perfusion of the heart with verapamil, a blocker of L-type calcium channels, changed the macro-dynamics of the heart between VT and VF. This study has revealed that verapamil perfusion consistently converts rapid pacing-induced VF to VT in isolated rat hearts, and characterized such verapamil-induced changes in the macro-dynamics of the heart. 


\section{Materials and Methods}

The animal experiments conformed to the "Principles of laboratory animal care" (NIH

publication No. 85-23, revised 1996), as well as the "guide for the care and use of laboratory animals”, Hokkaido University School of Medicine.

\section{Preparation of isolated heart}

The method of heart isolation was described elsewhere in detail $(5,15)$. In short, a total of 18 Male Wistar rats (8 weeks old, 240 - 260 g) were anesthetized with diethylether and administered heparin at $400 \mathrm{U} / \mathrm{Kg}$ intravenously. The chest was opened, the aorta cannulated, and the heart excised and immediately placed in ice-cold Krebs-Henseleit bicarbonate (KHB) buffer. The heart was then connected to the Langendorff apparatus and aortically perfused in a non-recirculating constant pressure $\left(90 \mathrm{cmH}_{2} \mathrm{O}\right)$ mode. The perfusate was KHB buffer containing (in mM) NaCl 120, $\mathrm{NaHCO}_{3} 25, \mathrm{KCl} 4.7, \mathrm{MgSO}_{4} 1.2, \mathrm{KH}_{2} \mathrm{PO}_{4} 1.2, \mathrm{CaCl}_{2}$ 1.2, and glucose 11. The buffer solution was equilibrated to a $95 \% \mathrm{O}_{2} / 5 \% \mathrm{CO}_{2}$ gas mixture of $\mathrm{pH} 7.4$ at $37^{\circ} \mathrm{C}$. The heart was enclosed in a small waterjacketed chamber. The temperature of the perfusate, as well as of the atmosphere surrounding the heart, was thermostatically controlled. 
A water-filled elastic balloon was inserted into the left ventricle via the left atrium. The

left ventricular pressure (LVP) was monitored by a pressure transducer (DT-XXED, Ohmeda, Madison, WI) connected to the balloon, and was continuously monitored and recorded. The initial value of the end diastolic pressure (EDP) was set to 7-10 mmHg by adjusting the volume of the balloon. Unipolar left ventricular cardiomyogram (LVCMG) recordings were made by implanting enamel-insulated copper wires ( diameter, $70 \mu \mathrm{m}$ ) into the left ventricular muscle. Unipolar LVCMGs were then fed to the band-pass filter $(0.08 \mathrm{~Hz}-3.0 \mathrm{KHz})$.

Induction of ventricular tachyarrhythmias

Hearts were perfused for 20 min with KHB solution (equilibrium period). The left ventricle was then electrically stimulated with a bipolar electrode inserted into the left ventricular muscle. Burst pacing (pulse interval, 40-70 ms (14-25 pulses/sec); pulse width, 1 ms; Intensity, 60-80 V) of the heart for 60 sec or more successfully induced a sustained VF or VT in most of the tested hearts (16 out of 18 hearts). Burst pacing usually induced a sustained VF, but in some cases a sustained VT. When burst pacing with the same pulse interval led to a non-sustained VF or VT, the pulse interval was automatically decreased stepwise in $5 \mathrm{~ms}$ 
decrements from 70 or $60 \mathrm{~ms}$ to $40 \mathrm{~ms}$ (programmed electrical stimulation protocol). The duration of each step was set at 20-30 sec.

Identification of arrhythmia

A digital storage-type oscilloscope (VC-11, Nihon Kohden, Tokyo, Japan) and a thermal pen recorder were used in the identification and analysis of waveforms and the diagnosis of arrhythmia. According to Merillat et al. (12), VF was defined as 1) the development of a chaotic, irregular rapid LVCMG, 2) the loss of pulsatile left ventricular pressure (LVP), and 3) the loss of grossly observable, regular ventricular contraction. Monomorphic VT was defined as a rapid LVCMG with a regular and stable rhythm and a regular cyclic change in LVP with small amplitude. The induced VT or VF was considered sustained if these conditions persisted longer than $5 \mathrm{~min}$.

Signal processing

The LVCMG signals stored on a DAT recorder were fed into an IBM compatible personal computer with "QuickVu-II” software (Teac, Tokyo, Japan), and analyzed on "Matlab” (The 
MathWorks, Inc., MA). Spectral analysis was performed using the fast Fourier transform

(FFT). Unipolar LVCMGs and LVP, in some cases, were used for the FFT analysis.

Drugs used and their application

Verapamil and nifedipine, blockers of L-type calcium channels, were obtained from Sigma

(St. Louis, MO). The other chemicals were from Wako Chem. (Tokyo, Japan).

Statistics

The data are expressed as the mean \pm S.D. Comparisons were performed using the one-way analysis of variance (ANOVA) followed by a paired t-Test. A $P$ value of less than 0.05 was considered statistically significant. 


\section{Results}

During pacing-induced sustained VF, we perfused the hearts with $600 \mathrm{nM}$ verapamil, a

blocker of L-type $\mathrm{Ca}^{2+}$ channels. Figure 1 shows an example of LVCMG, LVP, and the FFT spectrum from one heart treated with $600 \mathrm{nM}$ verapamil. The LVCMG before RR perfusion was irregular (1A \& 1a), with a characteristic VF-like wide-band spectrum (1d). The amplitude of LVP was small and irregular (1A \& 1a). Verapamil perfusion converted the VF to VT, as is seen from the change in the pattern of the LVCMG (1A \& 1b), and from the change in the FFT spectrum (1e). After the washout of verapamil, neither the LVCMG nor FFT spectrum returned to those seen before the start of verapamil perfusion during this observation period of about 3 min (1c \& 1f). A short-term Fourier transformation of LVCMG indicates the characteristic changes in the frequency spectrum with time (1B). Perfusion of hearts with 200, $600 \mathrm{nM}$ or $1.0 \mu \mathrm{M}$ similarly converted the rapid pacing-induced persisting VF to VT in all of the 8 tested hearts. Figure 1C shows a short-term Fourier transformation of LVP, reflecting the characteristic changes in the frequency spectrum with time similar to that of LVCMG. Perfusion of the heart with 1.0 $\mu \mathrm{M}$ nifedipine, a different blocker of L-type $\mathrm{Ca}^{2+}$ channels, also converted the pacing-induced sustained VF to VT ( $\mathrm{n}=2$ hearts; data not shown), suggesting that 
the verapamil-induced conversion of sustained VF to VT was due to the blocking of L-type $\mathrm{Ca}^{2+}$ channels by verapamil.

We then performed a cross-correlation analysis between LVCMG and LVP associated with verapamil-induced conversion of pacing-induced VF to VT. Figure 2 shows an example of the analysis. During pacing-induced sustained VF, both LVCMG and LVP showed irregular and chaotic patterns (2A1), and the cross-correlation coefficient between LVCMG and LVP was small (2B1). Verapamil (600 nM) perfusion converted VF-like irregular patterns to VT-like regular ones (2A2), and the coefficient became large (2B2). The VT-like regular patterns of LVCMG and LVP persisted even after the washout of verapamil (2A3), and the correlation remained high (2B3). Figure 2C shows the change in the peak-to-peak amplitude of the correlation coefficient between LVCMG and LVP with time. The correlation suddenly became high at about $40 \mathrm{~s}$ after the start of verapamil perfusion. This abrupt change in the correlation was associated with the transition from VF-like to VT-like patterns in LVCMG and LVP caused by verapamil perfusion. Figure 2D shows the statistical comparison between the correlation coefficient during verapamil-induced VT and that before the treatment (VF). The coefficient during VT was significantly larger than that during VF. 
Rapid pacing-induced persisting VF was often unstable, during which spontaneous transition to VT sometimes occurred (5). Therefore, the possibility that verapamil-induced conversion of pacing-induced VF to VT was due to an accidental spontaneous transition triggered by exchanging perfusion solution cannot be perfectly excluded. We thus investigated whether perfusion of hearts with verapamil resulted in the conversion of VF-like chaotic changes of LVP to VT-like regular patterns during rapid pacing ( $\mathrm{n}=2$ hearts). Rapid pacing of the heart with pulses of $40 \mathrm{~ms}$ intervals (25 pulses/s) consistently produced the stable VF-like chaotic changes in the amplitude of LVP for more than 5 min as is seen from changes in the LVP (Fig. 3A1) and their short-term Fourier transformations (3B1). During rapid pacing of the heart, $600 \mathrm{nM}$ verapamil was perfused for about $70 \mathrm{~s}$ (3A2 \& 3B2). Verapamil markedly reduced the amplitude of changes in the LVP, and gradually converted VF-like chaotic changes in the LVP to VT-like regular patterns as is seen from the change in the LVP (3g, 3h \& 3i) as well as from the FFT spectrum (3j, 3k \& 3l). Analysis on LVCMG was not possible because of the contamination of large stimulation artifacts in the unipolar recording of LVCMG. Expanded time records showed that changes in the amplitude of LVP were almost independent of the onset of stimulating pulses of $40 \mathrm{~ms}$ intervals (25 pulses/s) in the absence of verapamil 
(3d-3f \& 3g). During and after the perfusion of verapamil, only the spectrum component at 25

$\mathrm{Hz}$ became evident, although the power spectral density was low (3k, 3l). A short-term Fourier transformation of LVP also indicates the characteristic changes in the frequency spectrum with time (3B2). This result suggested that verapamil-induced conversion of VF to VT observed in Fig. 1 was not due to an accidental spontaneous transition.

We then investigated whether rapid pacing could induce sustained VF when the heart was pre-perfused with verapamil for longer durations before the application of pacing stimulus ( $\mathrm{n}=2$ hearts) (Fig. 4). After $1 \mu \mathrm{M}$ verapamil perfusion for $2 \mathrm{~min}$, the heart was then rapidly paced with pulses of $40 \mathrm{~ms}$ interval for 1 min (4A). Rapid pacing produced a sustained VT instead of VF after the end of the pacing stimulation (4A, 4a \& 4b). Figures 4c and 4d shows the characteristic change of LVP response to the stimulation pulse during the continued rapid pacing. The cyclic change of LVP was phase-locked to the stimulation pulse; that is, a 1:2 entrainment occurred (4C). In contrast, the LVP change was not phase-locked to the stimulation pulses (4d). The abrupt transition in the peak frequency in the FFT spectrum from 12.5 to about $18 \mathrm{~Hz}$ reflects this phenomenon (4e \& 4f), suggesting that the reentrant circuit independent of the stimuli was built at the time of this transition. During sustained VT 
observed after the end of pacing in the presence of verapamil, the peak frequency of about 18 $\mathrm{Hz}$ remained almost constant. However, the peak frequency gradually became low after the washout of verapamil (4h). A short-term Fourier transformation of LVP also indicates the characteristic change in the frequency spectrum of LVP during rapid pacing as well as during and after verapamil perfusion (4B).

We then investigated whether perfusion with verapamil during pacing-induced sustained VT produced any change in either the LVCMG or the LVP ( $n=4$ hearts) (Fig. 5). During pacing-induced sustained VT, perfusion of hearts with $1 \mu \mathrm{M}$ verapamil did not result in appreciable changes in the VT-like patterns of the LVCMG (5A, 5a-5c), the FFT spectrum(5d-5f), the short-term Fourier transformation of the LVCMG (5B), or the FFT spectrum (5g-5i) and the short-term Fourier transformation of the LVP (5C). This finding suggests that the activity of L-type $\mathrm{Ca}^{2+}$ channels was already being suppressed during sustained VT. However, $1 \mu \mathrm{M}$ verapamil treatment produced a gradual reduction in the peak-to-peak amplitude of the VT-like regular cyclic change in the LVP (5A \& 5a-5c). 


\section{Discussion}

The present study has demonstrated that perfusion of isolated hearts with either verapamil or nifedipine, blockers of L-type $\mathrm{Ca}^{2+}$ channels, consistently resulted in the conversion of rapid pacing-induced sustained VF to VT, and characterized the changes in macro-dynamics of the heart associated with the transition from VF to VT.

Merillat et al. (12) reported that an increase in cytosolic, global $\mathrm{Ca}^{2+}$ per se seems unnecessary for the initiation and maintenance of $\mathrm{VF}$, and that an increase of $\mathrm{Ca}^{2+}$ influx through L-type $\mathrm{Ca}^{2+}$ channel (slow $\mathrm{Ca}^{2+}$ channel) is essential. Many studies supporting the idea that changes in the activity of L-type $\mathrm{Ca}^{2+}$ channel are involved in the initiation and maintenance of VF have been published. Rapid ventricular stimulation in isolated rabbit hearts in the presence of verapamil, a blocker of L-type $\mathrm{Ca}^{2+}$ channels, results in the induction of a sustained VT, not VF (13). In addition, verapamil infusion abolishes ischemia/reperfusion-induced VF in isolated rat hearts (16). Verapamil also induces a gradual transition from burst pacing-induced VF toward stationary reentrant VT reversibly $(14,17) . \quad$ In addition, the flattening of the action potential duration (APD) restitution curve caused by the inhibition of L-type $\mathrm{Ca}^{2+}$ channel activity by verapamil has been shown to convert VF to VT in 
canine or porcine ventricles $(18,19)$. The slope of APD restitution is known to determine certain dynamical behavior of the heart that is relevant to the transition between VT and VF (20).

All these previous findings lead to the idea that changes in the $\mathrm{Ca}^{2+}$ entry through the L-type $\mathrm{Ca}^{2+}$ channel plays a critical role in the transition between VT and VF.

Verapamil, one of the class IV antiarrhythmic agents, is not selective for the myocardial L-type $\mathrm{Ca}^{2+}$ channel (16). Therefore, there is a possibility that verapamil-induced conversion of pacing-induced sustained VF to VT was caused by the other drug actions of verapamil. Drug actions of verapamil include alpha receptor blockade, sodium channel blocking, recruitment of collateral flow and bradycardia (20). However, Farkas et al. (16) concluded that verapamil's protective effects on ischemia-induced VF in conscious rats $(22,23)$ are mirrored by similar actions in Langendorff-perfused rat hearts, and appear to be mediated by blocking L-type $\mathrm{Ca}^{2+}$ channel. The present result that perfusion with nifedipine, a different blocker of L-type $\mathrm{Ca}^{2+}$ channels, also resulted in the conversion of sustained VF to VT (data not shown) seems to support this idea.

This study demonstrated that verapamil or nifedipine perfusion converted the rapid pacing-induced sustained VF to VT, suggesting that the activation of L-type $\mathrm{Ca}^{2+}$ channels is 
involved in the transition from VT to VF. Perfusion of isolated hearts with verapamil during pacing-induced sustained VT did not result in the any appreciable change in the VT-like regular patterns of LVCMG (Fig. 5). This result suggested that the activity of L-type $\mathrm{Ca}^{2+}$ channels was already being suppressed during VT, and the activation of L-type $\mathrm{Ca}^{2+}$ channels would be necessary for the transition from VT to VF. If so, then these findings have raised the question as to what mechanisms are responsible for the activation of L-type $\mathrm{Ca}^{2+}$ channels associated with the transition from VT to VF.

A recent ultrastructual study (24) has revealed that $\mathrm{Ca}^{2+}$ release in cardiac myocytes occurs preferentially in close proximity to mitochondria; the distance between the nearest sarcoplasmic reticulum (SR) T tubule junctions and mitochondria averages $37 \mathrm{~nm}$. In addition, Gathercole et al. (25) have shown that L-type $\mathrm{Ca}^{2+}$ channels are clustered in the surface plasma membrane overlying the junctional SR to secure the $\mathrm{Ca}^{2+}$-induced $\mathrm{Ca}^{2+}$-release (CICR) in cardiac myocytes. All these findings lead to the idea that the intracellular $\mathrm{Ca}^{2+}$ in the microdomains surrounding the mouth of L-type $\mathrm{Ca}^{2+}$ channels, SR and mitochondria would change due to the alteration of uptake of $\mathrm{Ca}^{2+}$ by SR and/or mitochondria, resulting in the changes of intracellular $\mathrm{Ca}^{2+}$ dynamics. It is well known that the activity of L-type $\mathrm{Ca}^{2+}$ 
channels is negatively regulated by an increase in intracellular $\mathrm{Ca}^{2+}\left(\mathrm{Ca}^{2+}\right.$-dependent inactivation of L-type $\mathrm{Ca}^{2+}$ channels) (26). These possibilities are now being investigated. 


\section{Acknowledgments}

This work was supported by a grant-in-aid for scientific research from the Ministry of

Education, Science, and Culture of Japan 12480256 and 14658277 to KK. 


\section{References}

1. Myerburg RJ, Castellanos A: Cardiac arrest and cardiac death. p 742. In Braunwald E (Ed):

Heart Disease: a textbook of cardiovascular medicine, Philadelphia, PA: Saunders, 1997

2. Samie FH, Jalife J: Mechanisms underlying ventricular tachycardia and its transition to ventricular fibrillation in the structually normal heart. Cardiovasc Res 50, 242, 2001

3. Nikolic G, Bishop RL, Singh JB: Sudden cardiac death recorded during Holter monitoring.

Circulation 66, 218, 1982

4. Pratt CM, Francis MJ, Luck JC, Wyndkham CR, Miller RR, Quinones MA: Analysis of ambulatory electrocardiograms in 15 patients during spontaneous ventricular fibrillation with special reference to preceding arrhythmic events. J Am Coll Cardiol 2, 789, 1983

5. Kawahara K, Takase M, Yamauchi Y: Increased vulnerability to ischemia/reperfusion-induced ventricular tachyarrhythmias by pre-ischemic inhibition of nitric oxide synthase in isolated rat hearts. Cardiovasc Pathol 12, 49, 2003

6. Koretsune Y, Marban E: Cell calcium in the pathophysiology of ventricular fibrillation and in the pathogenesis of proarrhythmic contractile dysfunction. Circulation 80, 369, 1989

7. Lakkata EG, Guarnieri T: Spontaneous myocardium calcium oscillations: are they linked to ventricular fibrillation? J Cardiovasc Electrophysiol 4, 473, 1993 
8. Billman GE: Ro 40-5967, a novel calcium channel antagonist, protects against ventricular fibrillation. Eur J Pharmacol 229, 179, 1992

9. Billman GE: Intracellular calcium chelator, BAPTA-AM, prevents cocaine-induced ventricular fibrillation. Am J Physiol 265, H1529, 1993

10. Boden WE, Krone RJ, Kleiger RE, Oakes D, Dwyer E, Miller JP, Abrams J, Copromilas J, Goldstein R, Moss AJ, the Multicenter Diltiazem Postinfarction Trial Research Group: Electrocardiographic subset analysis of diltiazem on long-term outcome after acute myocardial infarction. Am J Cardiol 67, 335, 1991

11. Hansen JF, the Danish Study Group on Verapamil in Myocardial Infarction: Treatment with verapamil during and after an acute myocardial infarction: A review based on the Danish verapamil infarction trials I and II. J Cardiovasc Pharmacol 18:suppl. 6, S20, 1991

12. Merillat JC, Lakatta EG, Hano O, Guarnieri T: Role of calcium and the calcium channel in the initiation and maintenance of ventricular fibrillation. Cir Res 67, 1115, 1990

13. Watanabe Y, Uchida H: Verapamil-induced sustained ventricular tachycardia in isolated, perfused rabbit hearts. Jpn Circ J 51, 188, 1987

14. Samie FH, Mandapati R, Gray RA, Watanabe Y, Zuur C, Beaumont J, Jalife J: A mechanism 
of transition from ventricular fibrillation to tachycardia; Effect of calcium channel blockade on the dynamics of rotating waves. Circ Res 86, 684, 2000

15. Kawahara K, Takase M, Yamauchi Y: Ruthenium red-induced transition from ventricular fibrillation to tachycardia in isolated rat hearts: possible involvement of changes in mitochondrial calcium uptake. Cardiovasc Pathol 12, in press

16. Farkas A, Qureshi A, Curtis MJ: Inadequate ischaemia-selectivity limits the antiarrhythmic efficacy of mibefradil during regional ischaemia and reperfusion in the rat isolated perfused heart. British J Pharmacol 128, 41, 1999

17. Watanabe Y, Gray RA, Mandapati R, Asano Y, Jalife J: Verapamil converts ventricular fibrillation into sustained monomorphic tachycardia in the isolated rabbit heart. Pacing Clin Electrophysiol 20, 1136, 1997

18. Riccio ML, Koller ML, Gilmour RFJ: Electrical restitution and spatiotemporal organization during ventricular fibrillation. Circ Res 84, 955, 1999

19. Swissa M, Qu Z, Ohara T, Lee MH, Lin SF, Garfinkel A, Karagueuzian HS, Weiss JN, Chen PS: Action potential duration restitution and ventricular fibrillation due to rapid focal excitation.

Am J Physiol 282, H1915, 2002 
20. Garfinkel A, Kim YH, Voroshilovsky O, Qu Z, Kil JR, Lee MH, Karagueuzian HS, Chen PS: Preventing ventricular fibrillation by flattening cardiac restitution. Proc Natl Acad Sci USA 97, 6061, 2000

21. Curtis MJ, Walker MJA: The mechanism of action of the optical enantiomers of verapamil against ischaemia-induced arrhythmias in the conscious rat. Br J Pharmacol 89, 137, 1986 22. Curtis MJ, Walker MJA: An improved pithed rat preparation: the actions of the optical enantiomers of verapamil. Asian Pacific J Pharmacol 1, 73, 1986

23. Curtis MJ: Calcium antagonists and coronary artery disease: an oppotunity missed? J Neural Transmission 31, 17, 1990

24. Sharma VK, Ramesh V, Franzini-Armstrong C, Sheu S-S: Transport of $\mathrm{Ca}^{2+}$ from sarcoplasmic reticulum to mitochondria in rat ventricular myocytes. J Bioenerg Biomem 32, 97, 2000

25. Gathercole DV, Colling DJ, Skepper JN, Takagishi Y, Levi AJ, Severs NJ: Immunogold-labeled L-type calcium channels are clustered in the surface plasma membrane overlying junctional sarcoplasmic retuculum in guinea-pig myocytes - implications for excitation-contraction coupling in cardiac muscle. J Mol Cell Cardiol 32, 1981, 2000 
26. Anderson ME: $\mathrm{Ca}^{2+}$-dependent regulation of cardiac L-type $\mathrm{Ca}^{2+}$ channels: is a unifying mechanism at hand? J Mol Cell Cardiol 33, 639, 2001 


\section{Figure Legends}

Fig. 1

Verapamil-induced conversion of pacing-induced sustained VF to VT. Perfusion of hearts with $600 \mathrm{nM}$ verapamil, a blocker of L-type $\mathrm{Ca}^{2+}$ channels, converted the pacing-induced sustained VF to VT reversibly (A). Figures a, b, and c in the lower panel in A show the expanded recordings indicated by a, b, and c in the upper panel of LVCMG, respectively. The LVCMG with a VF-like unstable pattern (a) changed to a VT-like stable LVCMG (b) caused by verapamil treatment. The LVP with a VF-like irregular pattern (a) also changed to a VT-like regular pattern (b). The period of verapamil perfusion is indicated by a bold bar under the recording of LVP in A. Figures d, e, and $\mathrm{f}$ indicate the power spectrum change of LVCMG during verapamil-induced conversion of tachyarrhythmia from VF to VT. An FFT analysis was performed on the LVCMG shown in A to characterize the changes in the frequency spectrum associated with the VF to VT conversion. d, e, f: FFTanalysis on LVCMG for 9 sec during the period indicated by bold bars (d, e, f) in B. B: short-term Fourier transformation of LVCMG every 1.5 sec. Power spectral density (PSD) increases from dark blue to red through 
yellow. Figures g, h, and i show the power spectrum change of LVP. g, h, i: FFTanalysis on LVP for 9 sec during the period indicated by bold bars (g, h, i) in C. C: short-term Fourier transformation of LVP every $1.5 \mathrm{sec}$. Power spectral density (PSD) increases from dark blue to red through yellow. Abbreviations: LVCMG, unipolar recording of left ventricular cardiomyogram; LVP, left ventricular pressure; PSD, power spectrum density.

Fig. 2

Changes in the cross-correlation function between LVCMG and LVP associated with verapamil-induced conversion of VF to VT. Figures A1, A2, and A3 show the LVCMG and LVP during before (A1), during (A2), and after (A3) verapamil (600 nM) perfusion, respectively. Figures B1, B2, and B3 show the cross-correlation coefficient between LVCMG and LVP before (B1), during (B2), and after (B3) verapamil perfusion, respectively. Figure C shows the changes in the cross-correlation coefficient between LVCMG and LVP with time. In this figure, the coefficient was calculated as follows: (mean peak-to-peak amplitude of the coefficient)/2.0. Figure D shows the statistical comparison between the correlation coefficient during verapamil-induced VT and that before the treatment (VF). Data are 
expressed as the mean+SD ( $\mathrm{n}=4$ hearts for VT or VF). $\quad * \mathrm{p}<0.05$.

Fig. 3

Verapamil-induced conversion of VF-like irregular change in the LVP to VT-like regular cyclic change during rapid pacing. The isolated heart was paced with repetitive pulses of 40 ms interval (25 pulses/s) for more than 5 min. The upper trace of A1 indicates the pacing-induced change in the LVP. During rapid pacing of the heart, the contamination of large stimulation artifacts made it difficult to perform an FFT analysis on the unipolar LVCMG recordings. Figures a-c show the expanded time records indicated by downward arrows (a, b, c) in the LVP recording. Figures d-f show the power spectrum of the LVP for 9 sec during the periods indicated by bold bars (d, e, f) in B1. Figure B1 shows a short-term Fourier transformation of the LVP during rapid pacing. Note that rapid pacing produced stable VF-like chaotic changes in the amplitude of LVP. Figures A2 and B2 indicate the verapamil-induced change in the LVP and the short-term Fourier transformation of it during rapid pacing, respectively. The isolated heart was paced with repetitive pulses of $40 \mathrm{~ms}$ interval, and then perfused with $1.0 \mu \mathrm{M}$ verapamil. Figures g, h, and i show the expanded time recordings 
during the periods indicated by downward arrows (g, h, i) in the LVP recording. Figures j, k, and $\mathrm{l}$ show the power spectrum for 9 sec during the periods indicated by bold bars $(\mathrm{j}, \mathrm{k}, \mathrm{l})$ in B2.

Abbreviations: stim, pulses of rapid pacing. Other abbreviations are the same as in Fig. 1.

Fig. 4

Rapid pacing-induced sustained VT caused by pre-treatment with verapamil. After 2 min verapamil (1.0 $\mu \mathrm{M})$ perfusion, the heart was then paced with repetitive pulses with $40 \mathrm{~ms}$ interval. The rapid pacing evoked sustained VT as is seen from the LVCMG and LVP (A \& a). After the washout of verapamil, the peak-to-peak amplitude of LVP gradually became larger, and the cycle period became longer (b). Figures e, f, g, and h indicate the power spectrum change of LVP. An FFT analysis was performed on the LVCMG shown in A to characterize the changes in the frequency spectrum. e, f, g, h: FFT analysis on LVP for 9 sec during the period indicated by bold bars (e, f, g, h) in B. B: short-term Fourier transformation of LVP every 1.5 sec. Power spectral density (PSD) increases from dark blue to red through yellow. See text for details. 
Fig. 5

Verapamil perfusion during pacing-induced sustained VT did not produce any appreciable change in the pattern of LVCMG. During rapid pacing-induced sustained VT, 1 $\mu \mathrm{M}$ verapamil was perfused for about 2 min. Verapamil perfusion did not result in the any marked change in the LVCMG pattern, but reduced the amplitude of the regular cyclic change in the LVP (A, a, b, \& c). Figures a, b, and c show the expanded time records indicated by downward arrows (a, b, c) in the LVCMG recording. Figures $d$, e, and $f$ show the power spectrum of the LVCMG, also indicating that verapamil perfusion does not produce any marked change in the spectrum. d, e, f: FFT analysis on LVCMG for 9 sec during the period indicated by bold bars (d, e, f) in B. B: short-term Fourier transformation of LVCMG every 1.5 sec. Power spectral density (PSD) increases from dark blue to red through yellow. Figures g, h, and i show the power spectrum of the LVP, indicating that verapamil perfusion does not produce any marked change in the spectrum. g, h, i: FFT analysis on LVP for 9 sec during the period indicated by bold bars (g, h, i) in C. C: short-term Fourier transformation of LVP every 1.5 sec. Power spectral density (PSD) increases from dark blue to red through yellow. 
LVP

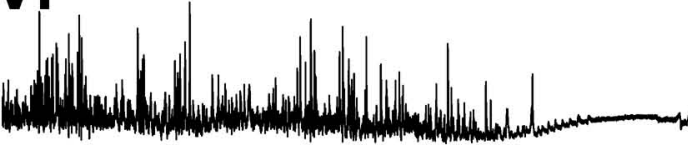

verapamil

a

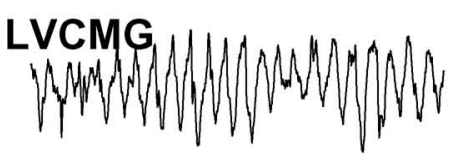

LVP

b

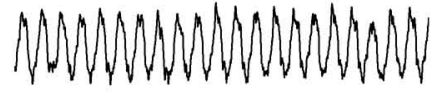

L

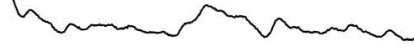

C

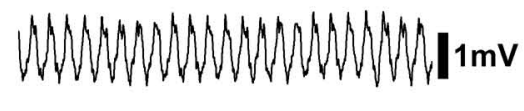

$200 \mathrm{~ms}$

$5 \mathrm{mmHg}$

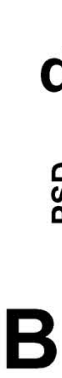

$\times 10^{8}$

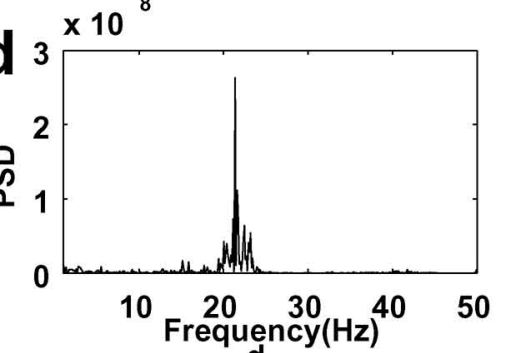

논
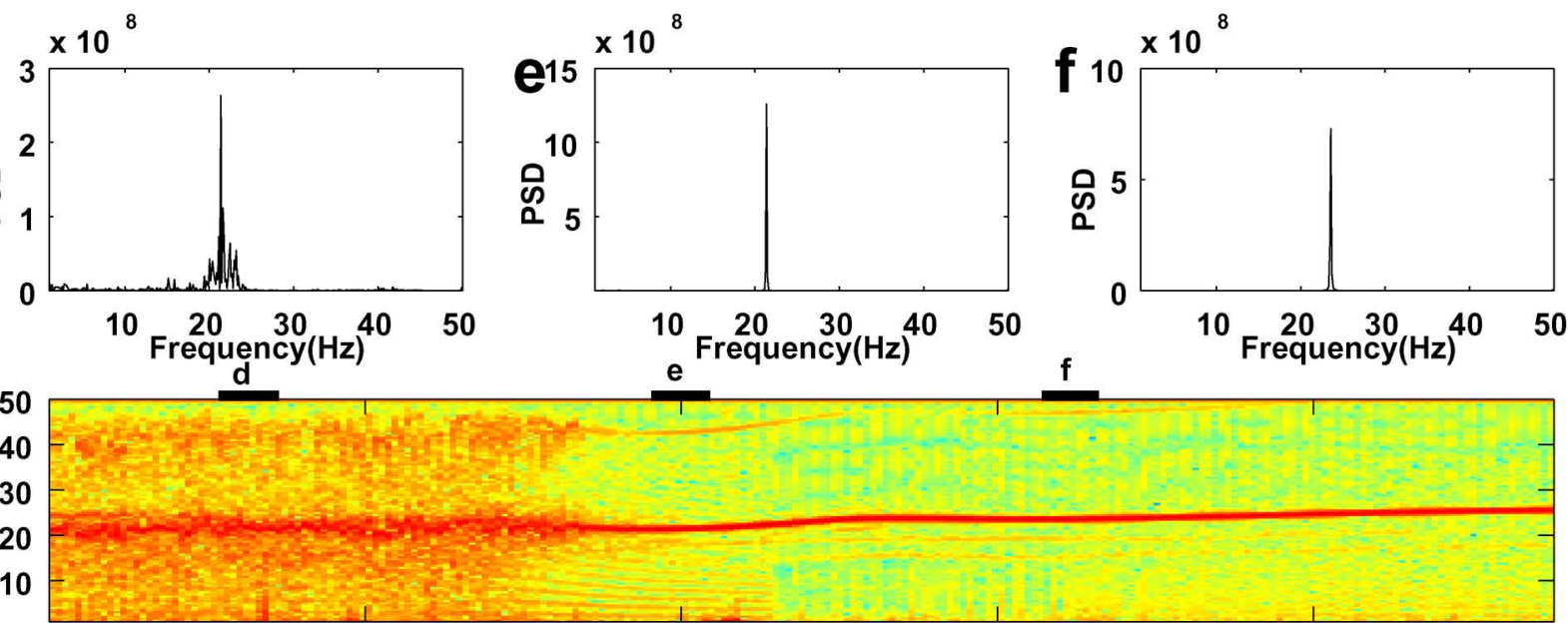

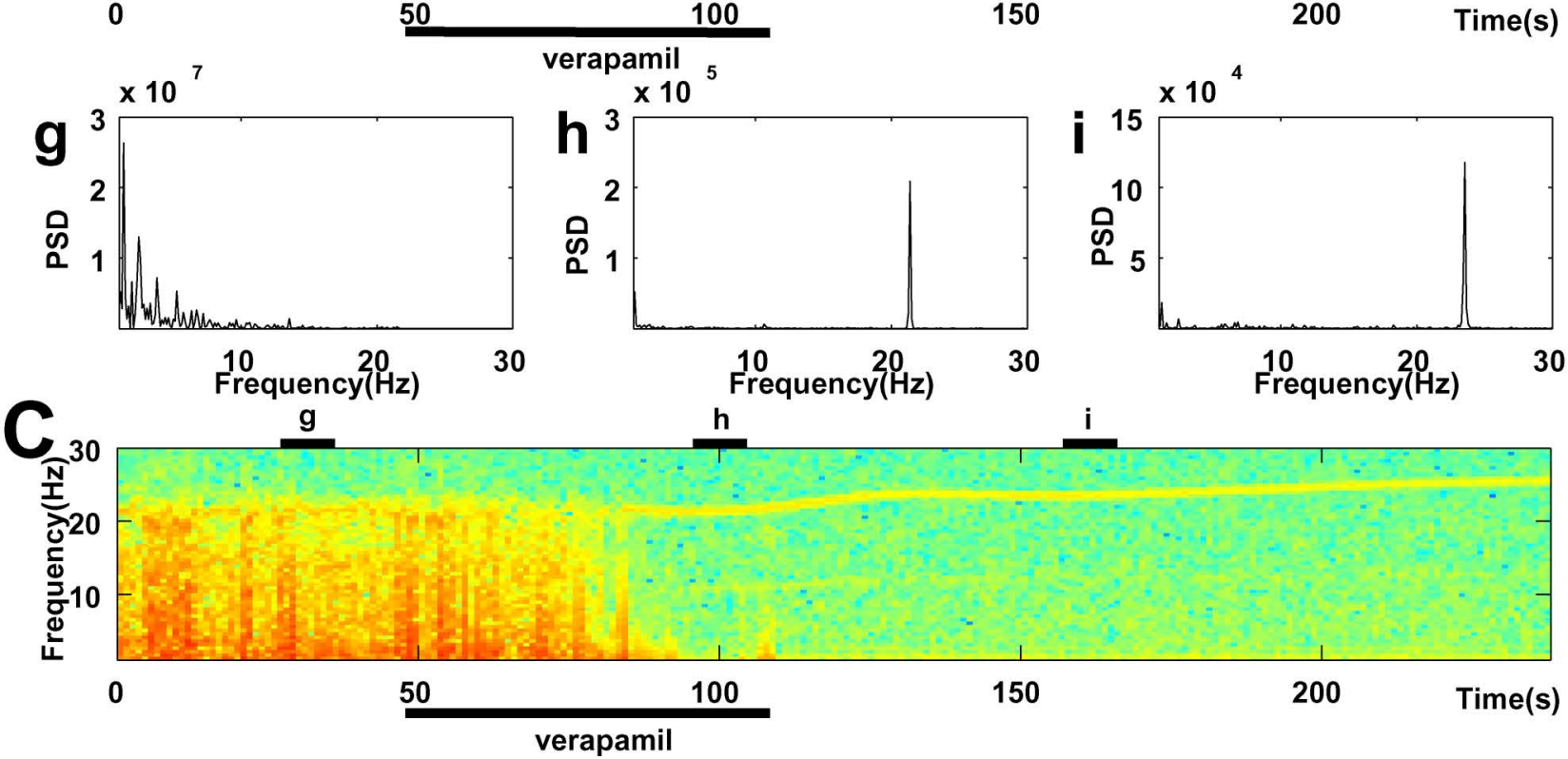



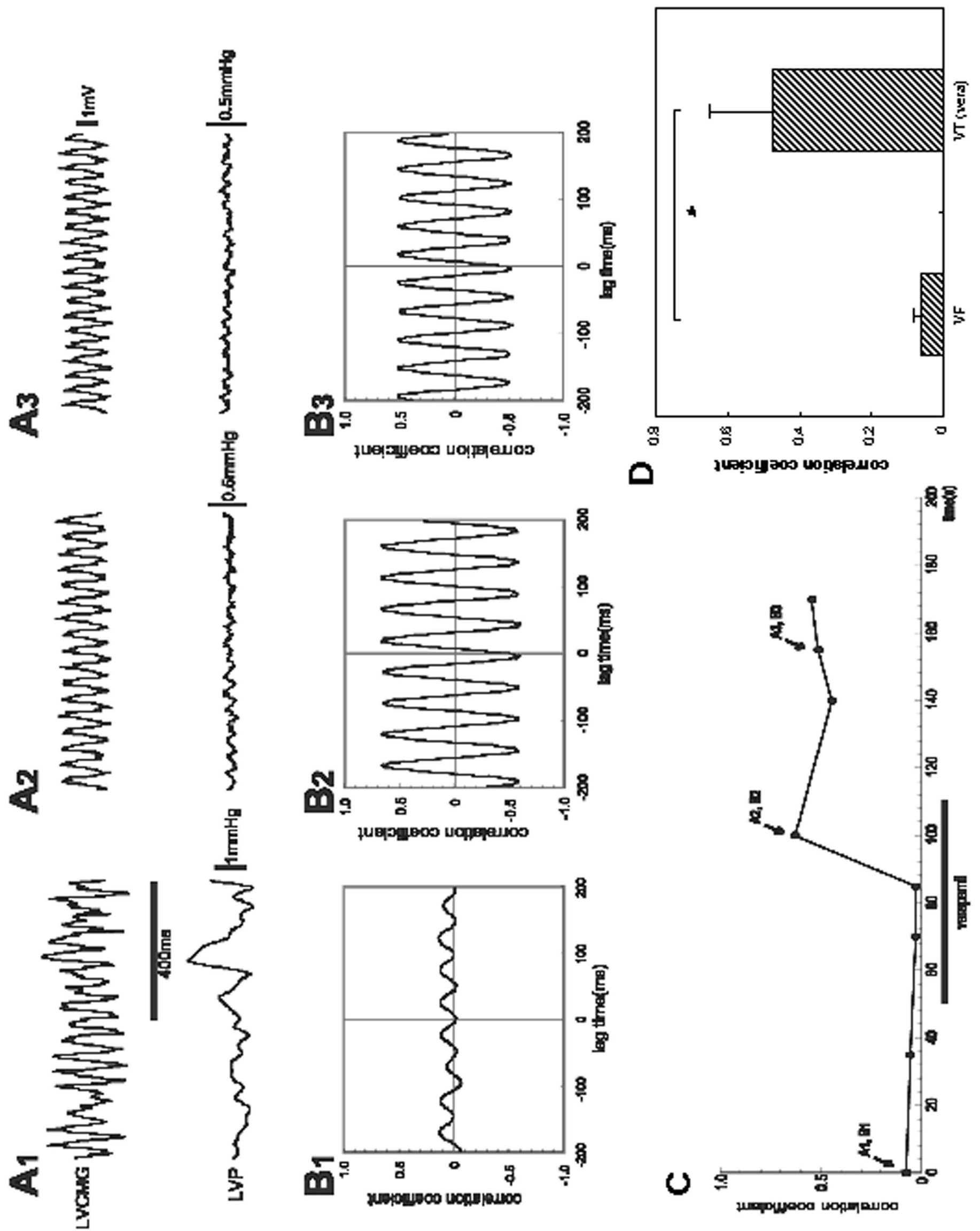

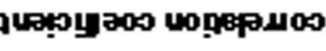

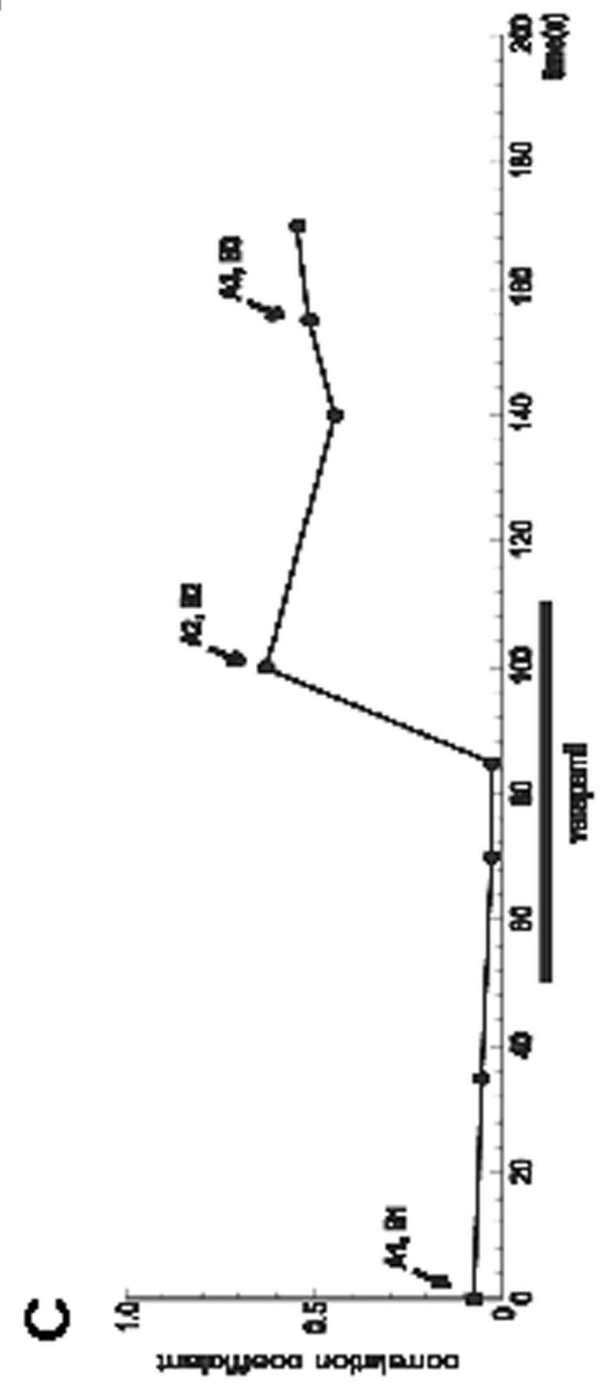


a

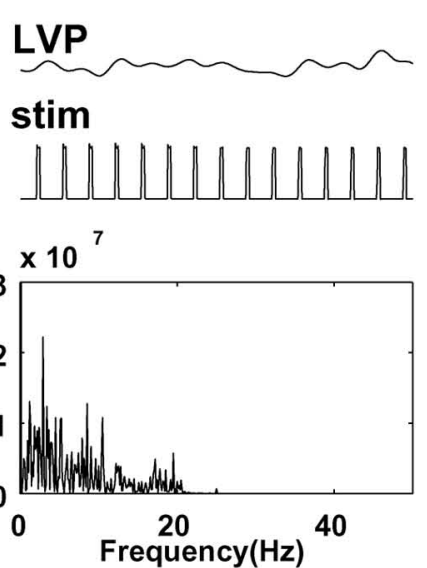

pacing
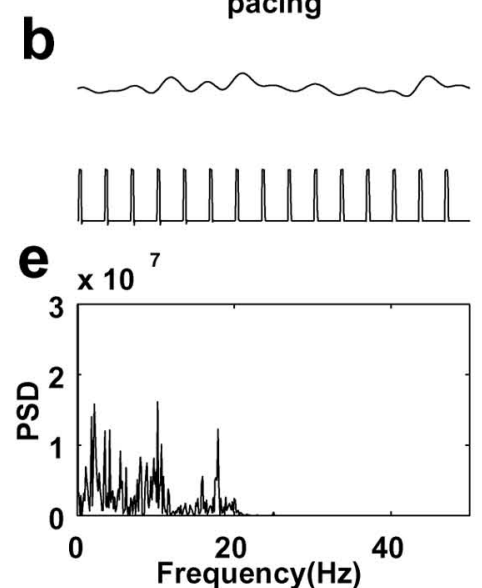
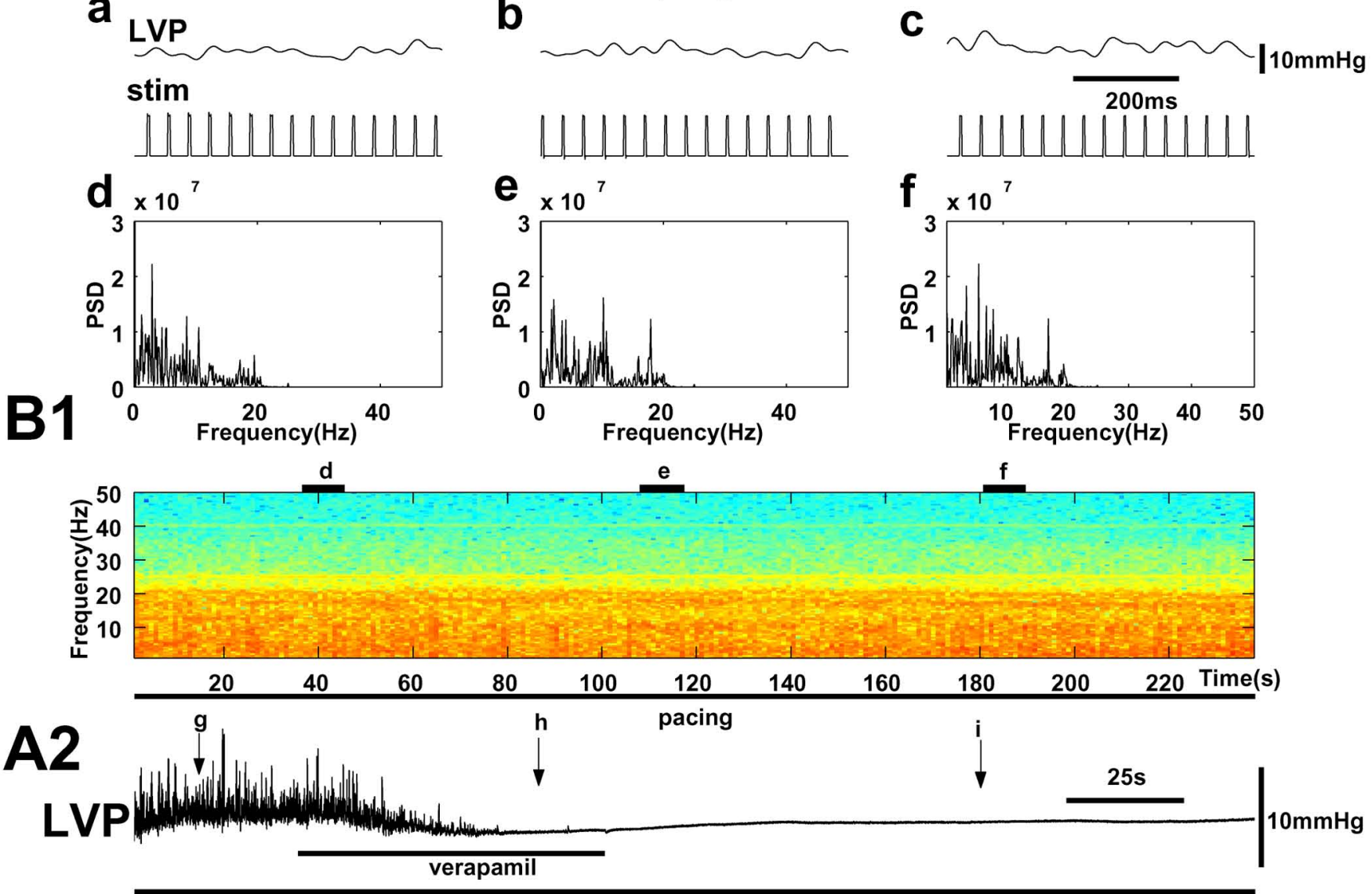

g LVP $M$ Mn

stim
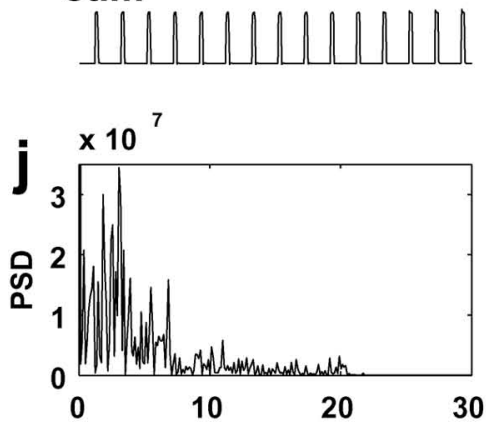

B2 h

pacing
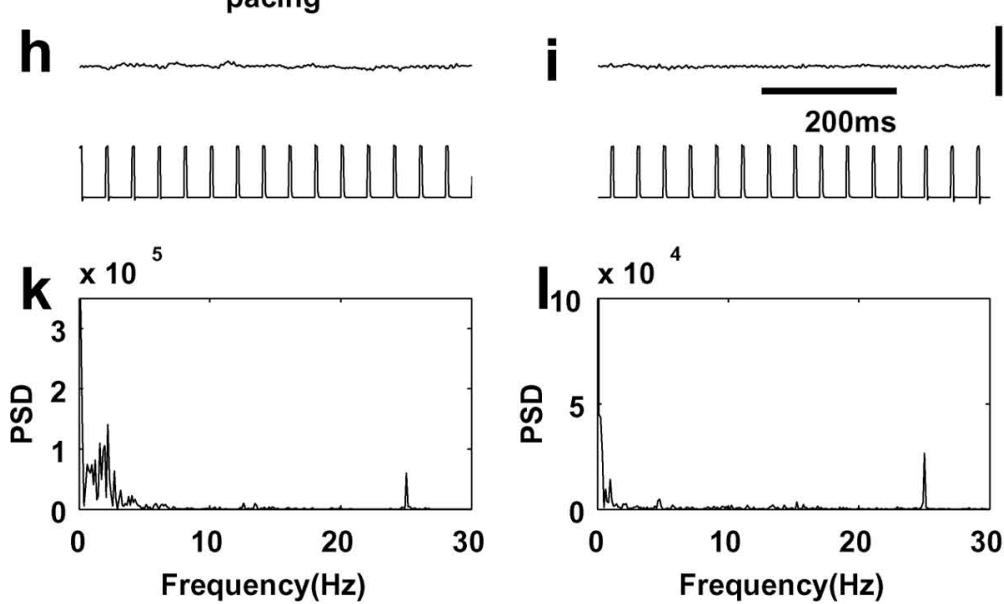

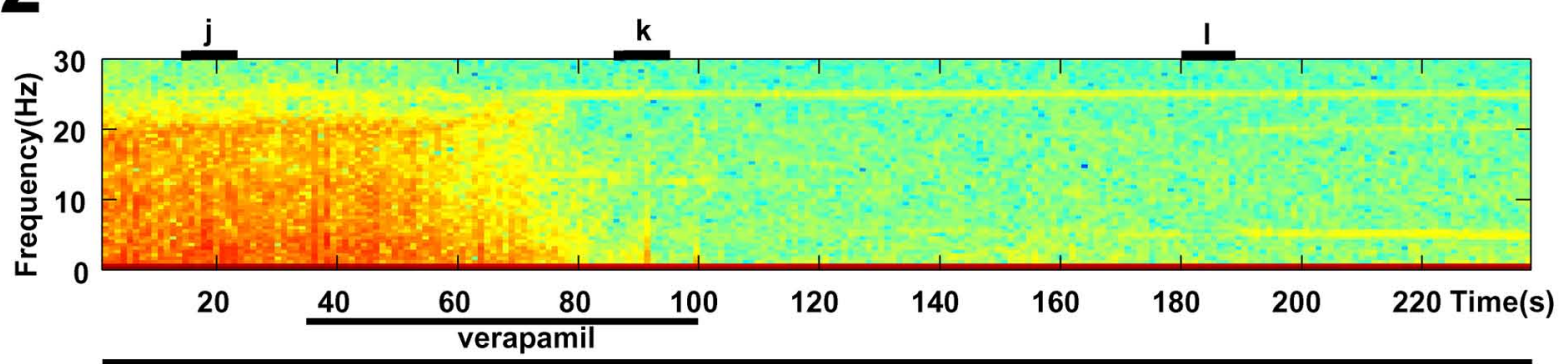

pacing 

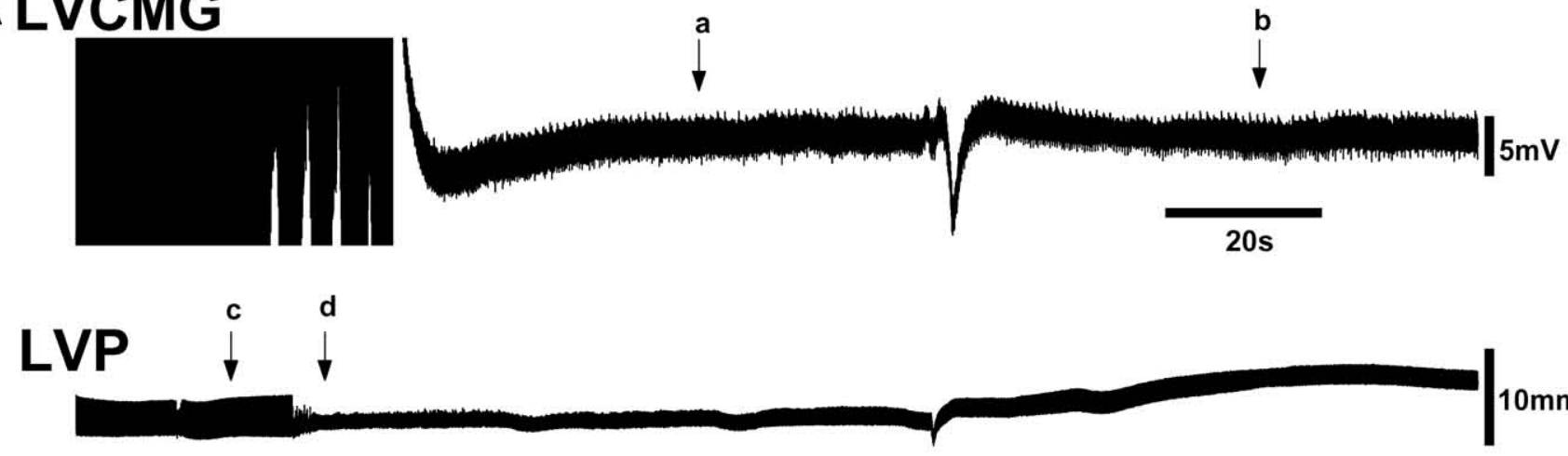

\section{verapamil}

pacing

a LVCMG

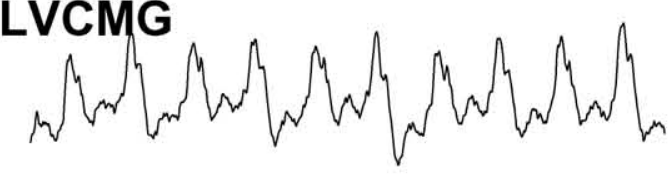

LVP

C

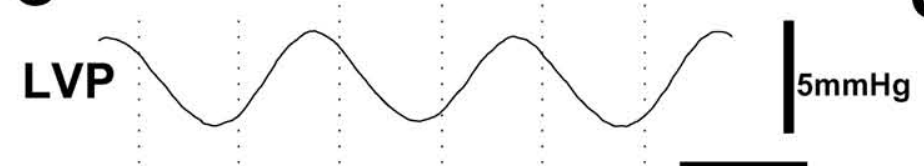

stim
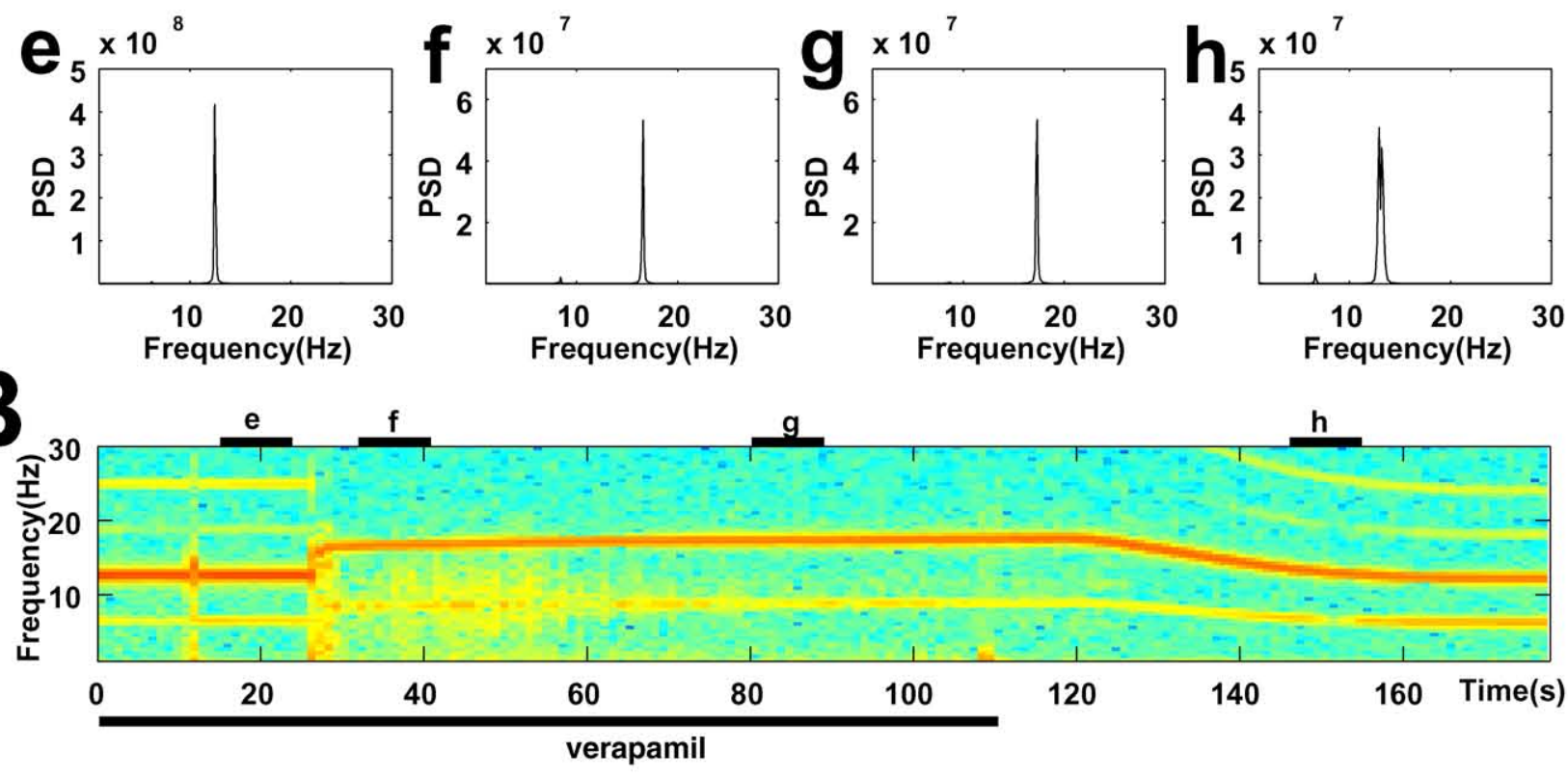

pacing b Whowhinhwhram

\section{0ms}

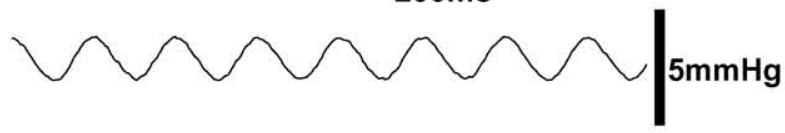

d

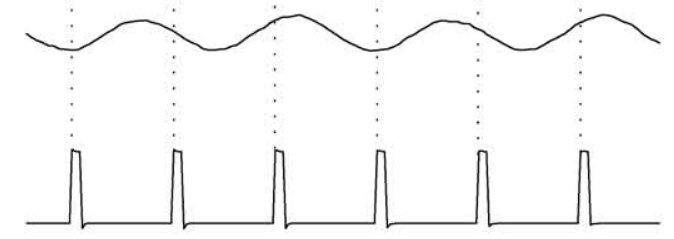


LVP

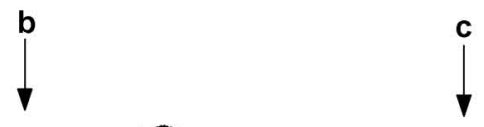

$2 \mathrm{mV}$

$25 \mathrm{~s}$

verapamil

$a_{\text {LVCMG }}$

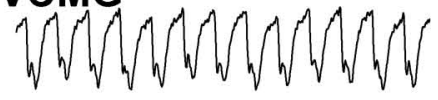

\section{LVP}

WWWWW

B

d

$10 \times 10^{9}$

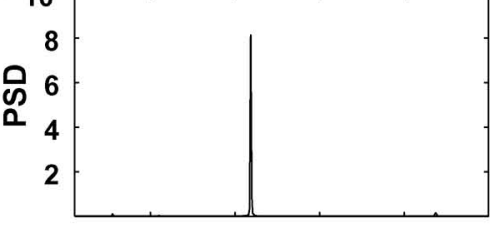

$\begin{array}{lllll}10 & 20 & 30 & 40 & 50\end{array}$ Frequency $(\mathrm{Hz})$ b

$W W W W W W W W W W W W$

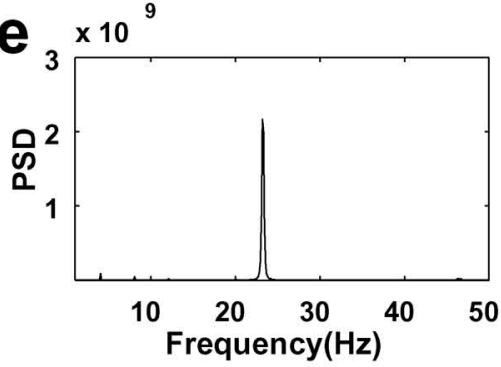

C

WWWWWWWWWWWWWlimv $250 \mathrm{~ms}$

mum $\left.\right|_{2 \mathrm{mmHg}}$

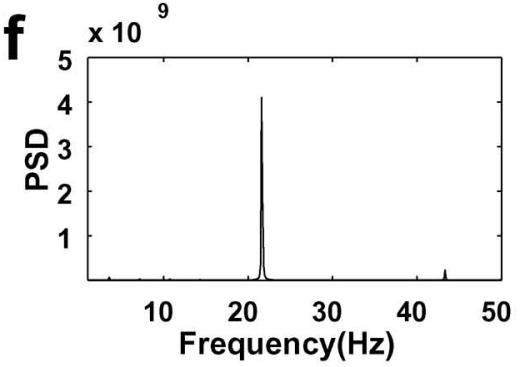

$\widehat{N}^{50}$
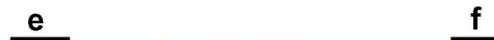

동

30

20

10

0

$$
50
$$

150

200

Time(s)

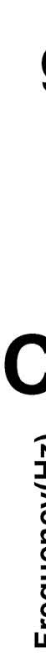

verapamil $_{6}$

h

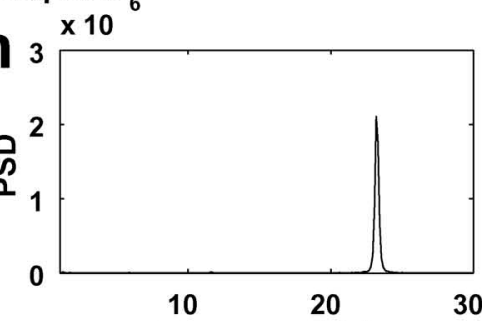

Frequency $(\mathrm{Hz})$

$h$

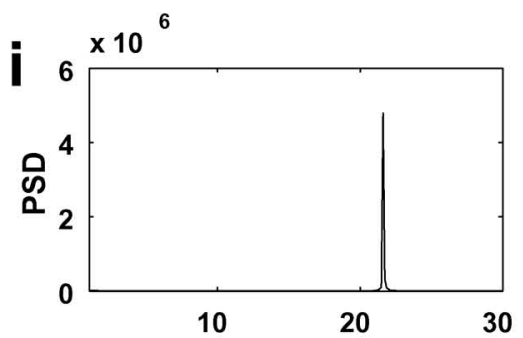

Frequency $(\mathrm{Hz})$

cy $(\mathrm{Hz})$ g

.
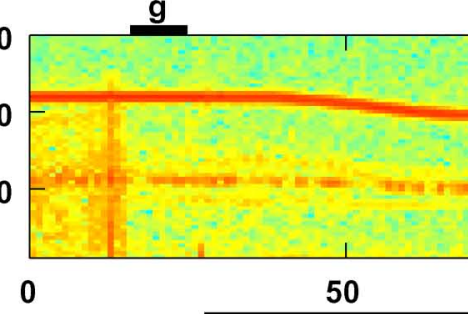